\title{
Robotics as a support tool for experimental optimisation of surgical strategies in orthopaedic surgery
}

\author{
Manel Frigola ${ }^{\mathrm{a}}$, Manuel Vinagre ${ }^{\mathrm{a}}$, Alicia Casals ${ }^{\mathrm{a} *}$, Josep Amat ${ }^{\mathrm{b}}$, Fernando Santana ${ }^{\mathrm{c}}$ and Carlos Torrens ${ }^{\mathrm{c}}$ \\ ${ }^{a}$ Institute for Bioengineering of Catalonia and Technical University of Catalonia, Baldiri Reixac, 08028 Barcelona, Spain; ${ }^{b}$ Department \\ of Automatic Control, Technical University of Catalonia, Edifici Omega, Jordi Girona, 1-3, Barcelona, Spain; ' Orthopedic Department \\ at Hospital del Mar, IMAS, Passeig Maritim, 08003, Barcelona, Spain
}

(Received 19 February 2010; final version received 14 June 2010)

\begin{abstract}
Robotics has shown its potential not only in assisting the surgeon during an intervention but also as a tool for training and for surgical procedure's evaluation. Thus, robotics can constitute an extension of simulators that are based on the high capabilities of computer graphics. In addition, haptics has taken a first step in increasing the performance of current virtual reality systems based uniquely on computer simulation and their corresponding interface devices. As a further step in the field of training and learning in surgery, this work describes a robotic experimental workstation composed of robots and specific measuring devices, together with their corresponding control and monitoring strategies for orthopaedic surgery. Through a case study, humerus arthroplasty, experimental evaluation shows the possibilities of having a test bed available for repetitive and quantifiable trials, which make a reliable scientific comparison between different surgical strategies possible.
\end{abstract}

Keywords: surgical robotics; training robotics; optimisation of surgical procedures; surgical techniques evaluation

\section{Introduction}

Current surgical practice shows that some kinds of orthopaedic interventions rely on procedures that are performed in a highly intuitive way, based on the knowledge of human anatomy and medical praxis. In such interventions, the lack of precise measures and anatomical references, as well as the differences between patients, hinder, in many cases, the establishment of a methodical and optimal procedure in which all the involved actions can be precisely quantified.

Under these circumstances, virtual reality and robotics can help supply tools to simulate and emulate surgical procedures and therapies in a scientific and rigorous way. The possibility of parametrically quantifying actions and movements can lead to a more objective and measurable evaluation process, as well as provide a way towards a better understanding and learning of human anatomy. Thus, the different application fields in which robotics and virtual reality can assist in surgery are assistance along an intervention, experimentation on new techniques and procedures, and surgical training. Enormous efforts have been devoted to robot assistance during an intervention, either to achieve some automated actions or to use any teleoperation mode. Much research has also been invested in surgical simulators for planning and training. However, much less activity has been dedicated to the design of robotic systems oriented to simulate an intervention using a physical device, and not only relying on a computer-based simulation program.

Simulation and virtual reality constitute excellent means of modelling 3D bones and soft tissues, achieving quite good results and providing a tool for first systems' evaluation. The main limitation of simulation is the reliability of the models that can be achieved when dealing with uncertainty on the characteristics of the objects to be simulated. In addition, and in contrast with the good performance attainable from visual and even haptic feedback when high-quality models of the working environment are available, an important drawback of simulation appears when dealing with some kinds of training operations that require dexterous manual operations. That is to say, in a training phase, in which an interaction of a professional trainer with the physical world is necessary, one such computer tool can turn out to be of little assistance. Therefore, the availability of an alternative solution based on some kind of mechanical device or robotic station becomes necessary.

Although the implementation of a robot-assisted physical simulator has the main drawback that designing and building a new workstation for each kind of surgery is needed, its advantages are manifold. Firstly, an intervention procedure, or part of it, can be evaluated in conditions similar to the real ones and health professionals can perform

\footnotetext{
*Corresponding author. Email: alicia.casals@upc.edu
} 
their training in highly realistic operating conditions, either using physical body models designed ad-hoc, or operating over human specimens. Secondly, the performance of robotic systems, in what refers to their operation in a repetitive and systematic way, makes it possible to quantitatively analyse and compare different operational strategies to determine an optimal solution. Finally, robotics has also proved to be a useful tool for understanding the 'why' of some solutions of the human anatomy.

In this latter case the study of human anatomy or its performance using robotic systems has already been used in other fields and it has proved to be an efficient means of understanding anatomical behaviour. Robotics also provides a source of information for the designing of better therapies or surgical procedures. Several robots or robotic structures have been designed with this aim. Takanobu et al. (1998) designed a masticator robot to quantify masticator efficiency, comparing clenching and grinding, by defining a quantification index for that study. Later on, a new biomechanical model of the masticator system was modelled mathematically in Xu et al. (2005). In a similar context, an elastic tongue mechanism was designed to model how human speech is produced (Nishikawa et al. 2003). Also referring to the study of the human body, models of the human spine, used originally to determine the best structure and location of implants, led to the designing of humanoid robots with human-like flexible spines (Mizuuchi et al. 2002).

Referring to the surgical training and performance evaluation, one of the problems found is linking visual and kinaesthetic information to obtain objective criteria for effective actuation. With this aim and to overcome the limitations of the sensors used commonly to relate this information, Rosen et al. (2002) used a robotic system. A two four-bar mechanism attached to the endoscopic tools replaces such sensors, thus avoiding the occlusions produced with the use of optic or acoustic sensors, or the interferences that affect electromagnetic measures when tracking the orientations of surgical tools in minimally invasive surgery (MIS). A study on the adequate design of such robotic devices for laparoscopic surgery is described by Zahraee et al. 2009.

As an aid in training, in Fuhan Shi and Payandeh (2007) a sutures simulator (finite elements computing) reproduces the behaviour of a real thread, modelled as a mass-spring system, in operations for knotting surgical sutures. The simulator is used to estimate not only the external and internal forces, including the friction force, produced by knotting and unknotting movements but it can also show how forces propagate along a suture when the user pulls it.

Conscious of the missing properties of computer simulators, Solis et al. (2009) describe a 'patient robot' which embeds sensors and actuators into a conventional human model. A first approach to this goal was the development of a trainer composed of a skinny dummy, an array of embedded sensors to acquire quantitative data while performing surgical tasks, a webcam and a personal computer. From the experimental results, the system proved to be an efficient tool for training as it was able to detect differences among different levels of skill.

In Wang et al. (2006) both the patient and the robot are simulated by means of finite element models in a training station designed to learn the use of surgical robots.

This work focuses on the developing of tools for training and learning. It is not addressed to tackle a concrete surgical technique, but toward the design and implementation of a robotic environment that allows the physical experimentation of surgical techniques that rely on the use of robotics at different levels, ranging from auxiliary or evaluation functions (robot as a measuring instrument) to executive functions (such as cutting or knotting). These robotic operations can be performed in a master-slave configuration, or even automatically, if the task is planned with precise pre-defined models.

\section{Characteristics of an experimental work station}

The progress of medical and surgical practice is the result of three basic factors: the study of basic knowledge accumulated by the experience gained from regular surgery, a better learning and training process and, finally, from experimentation with new materials and procedures. The continuous feedback between scientific contributions and medical practice is a key factor in this progress.

Thus, the achievement of new improvements does not uniquely depend on daily practice, but on the availability of more powerful and flexible scenarios designed for the experimentation and evaluation of surgical techniques. This concept is shown in Figure 1, which shows the confluence of experience gained from regular surgical practice, either operating manually or through the use of robotic systems, experience acquired through the use of simulators and experience and abilities trained and reinforced using physical experimental stations. One such experimental station can be composed of hardware and software components. In this work, experimentation is considered in its broad sense, comprising simulation in virtual environments, operation over specimens and, finally, relying on a robotised station constituting a physical emulation of a patient, or part of it.

The proposed experimental robotic environment has been conceived in such a way that it can support a wide variety of experimental works. For this reason, the developed workstation has been endowed with the capability of actuation and monitoring of either automatic operation or tutelary tasks. The automatic execution mode is focused on interventions in which rigidity and anatomical stability allow the surgeon to plan the intervention based on 3D models obtained from computerised tomography (CT) images. In the tutelary or supervised mode the surgeon manually performs the intervention in cooperation with a robot, which is the final real executor. The surgeon's manual guidance 


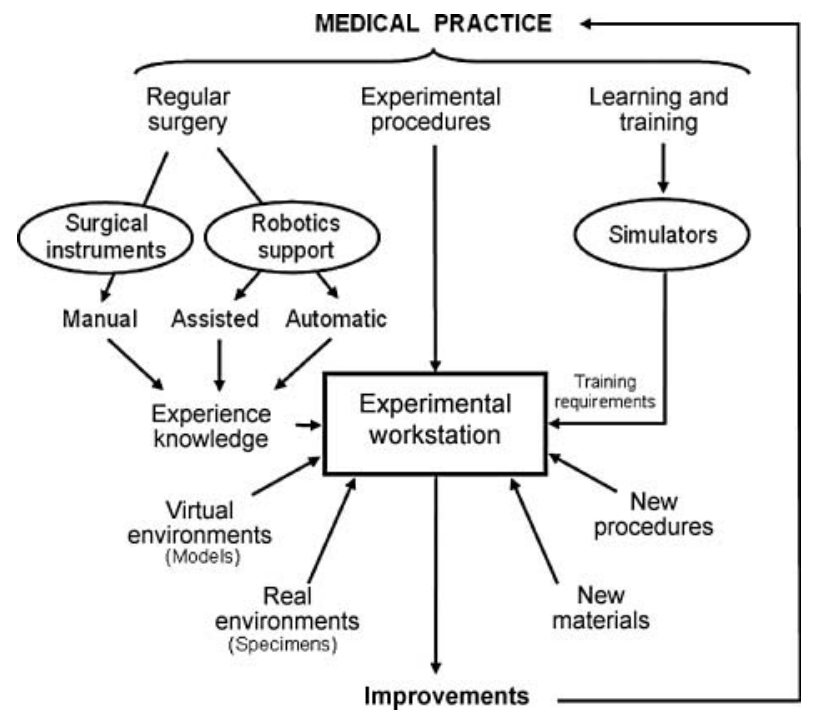

Figure 1. Schema of the items involved in the improvement of surgical robotics.

can be performed either in situ, or from a distant master location. The resulting robot actuation is carried out within the constraints that the surgeon has fixed previously.

This platform is oriented to quantitatively evaluate the surgical quality achieved from operations carried out on biomechanically equivalent models, in which the appropriate mechanical assays are conducted and monitored. The following sections describe the configuration of the implemented experimental station. The functions of the workstation are described in Section 2, together with an explanation of the sensory structure and characteristics. Then the study of the system performances is studied in Section 3, tackled from the medical perspective and defining an evaluation criterion. After analysing the obtained results the article concludes with some conclusions and discussion on the achieved medical improvements and knowledge gained.

\subsection{Configuration of the workstation}

The developed workstation consists of a test bed in which some mechanical parameters, such as efforts, torques or displacements, or temporal behaviours, such as tiredness or deterioration, can be evaluated using adequate sensors. A functional schema of this platform is shown in Figure 2.

In order to be able to adapt to the requirements of the above-mentioned operation modes in orthopaedic surgery, it is necessary to measure the required parameters and execute some given movements. For this reason, the developed workstation is composed of two robot arms that can be programmed to execute repetitive trajectories and monitoring tasks and tests. It can operate either in teleoperation mode by means of remote master devices, handheld-type devices or sensitised gloves, or in comanipulation mode by means of force-torque-based control strategies (Figure 3). The two

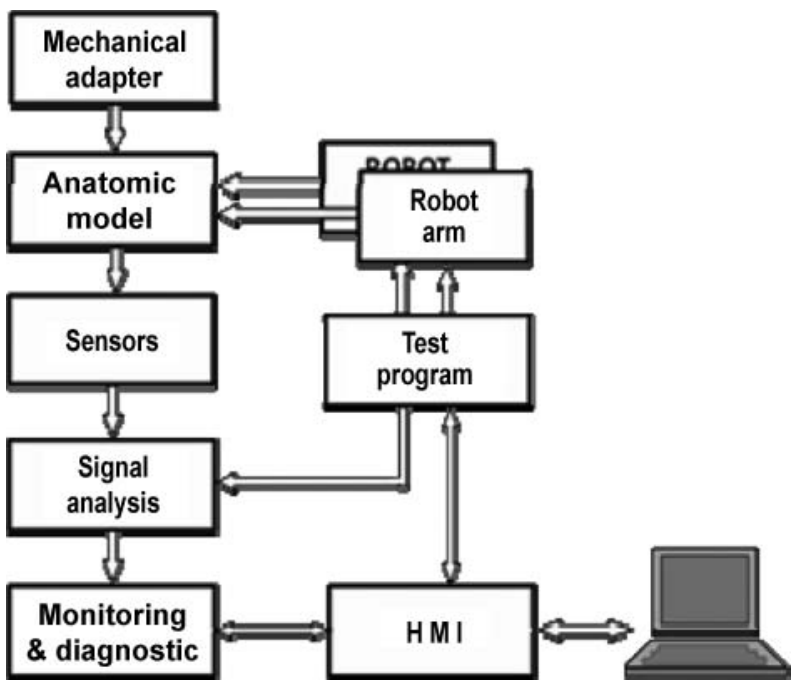

Figure 2. Functional schema of the experimentation platform.

six-degree-of-freedom (6-DoF) commercial robots, Staubli RX160s, are endowed with six-DoF force and torque sensors, ATI, at their wrists.

The experimental platform is endowed with a computer provided with a signal acquisition system for the evaluation of the surgical quality achieved in each procedure subjected to test and evaluation. From the sensor signals acquired, an analysis and evaluation of the results can be done either in an absolute way or statistically.

The execution of tutelary operations, that is with the cooperation of the human and the robot, carries with it some requirements, such as having more sensory capabilities available. The operational sensor data is necessary to be able to feed the required haptic information back to the surgeon so that he or she can cooperate efficiently.

The need to extract information, for monitoring and for the analytical evaluation of surgical strategies, has also forced us to consider a series of auxiliary elements with sensors to measure and monitor forces, torques and strains in rigid elements or in suture threads, as well as 3D and $6 \mathrm{D}$ displacements in space. Complementarily, other physiological sensors, such as tissue oxygenation sensors, can be added to the station so that their data can be correlated with those obtained from mechanical experimentation. With the measure of these parameters and with the execution of strictly repetitive trials, it has been possible to objectively compare materials or techniques, either on different kinds of identical physical models, or on specimens. In the latter case, working with cadavers, the anatomical trials can be unquestionable from the anatomical point of view; however, such trials are not suitable for comparative analysis with strict repetitive conditions. In each trial, the specimen is modified somehow during the experiment and it is not reusable for further tests in the same working conditions. 


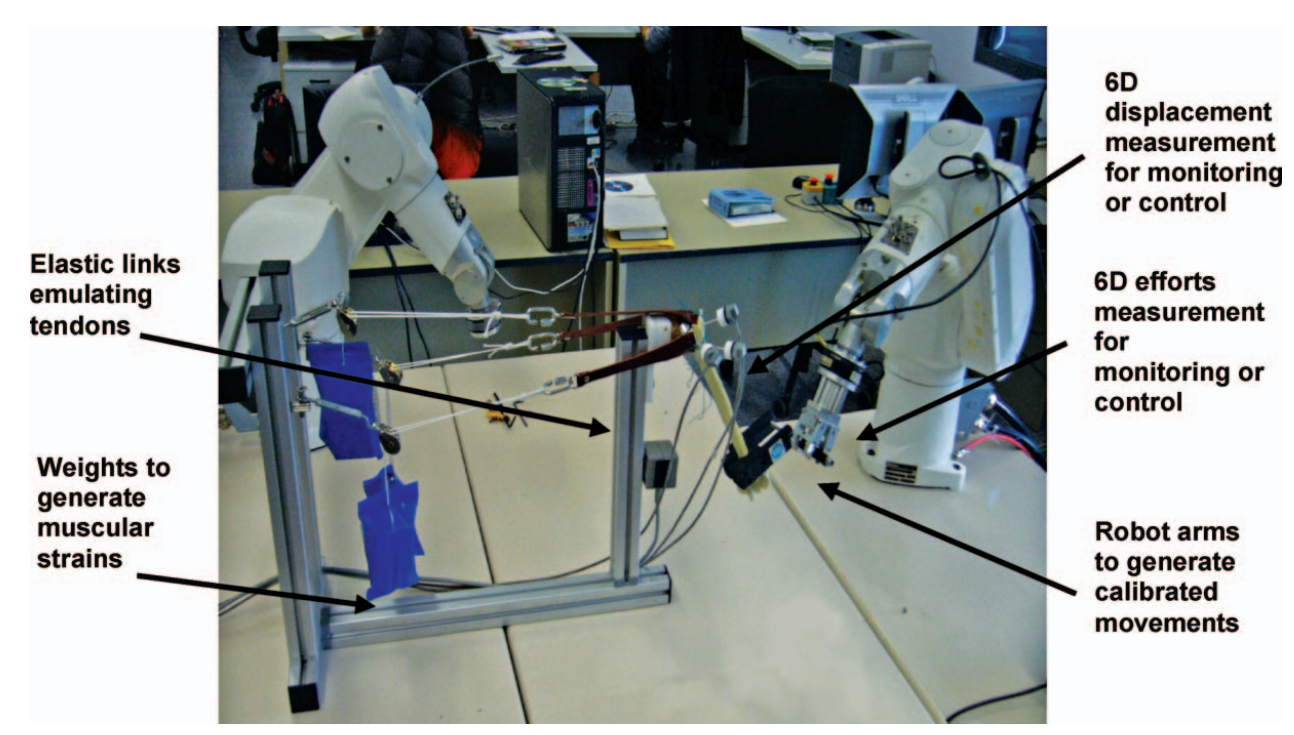

Figure 3. The experimental sensitised and robotised workstation.

\subsection{Parameters measurement}

The two kinds of measure considered above are mainly related to soft and hard tissues respectively. Tissue regeneration and correct bone fixation are some of the main concerns in orthopaedic surgery. These factors strongly depend on correct suture placement and suture tension adjustment. Tight sutures may impede irrigation, loosely stretched sutures hinder tissue integration and/or bone fragments may rest in an unstable way. The design of a device that can measure the tensions supported by the sewing threads is not a trivial issue because the measuring device has to provide the value of each force without modifying either the tension or the geometry of the sewing threads as they are usually executed in surgery. Therefore, in order to monitor suture tensions, some devices have been developed (Shubert et al. 2006; Kiriyama et al. 2008) to validate surgical techniques such as MIS.

The solution proposed in this workstation for measuring strains considers two types of sensors (Figure 4). The first consists of a measurement frame endowed with a roller in such a way that the working strain of each sewing thread can be fixed with a screwdriver. Force measurement is based on strain gauges placed over this frame and the efforts are monitored under different working conditions (Figure 4c). The device consists of a metallic frame leaning on the two pieces of bone to be sutured. The adjustment to the roller provides the sewing thread with the tension equivalent to the one that surgeon normally performs in an intervention. The second type of sensors consists of grippers that measure the strain of the thread that is tensed by means of a knot performed by the surgeon (Figure 4d). These sensors should allow the measurement and monitoring of the strain of the thread with which the surgeon has fixed each sutur- ing point, thus constituting a useful tool for training without modifying the usual geometry of each suture.

In order to know the range of forces supported by each tendon during the rehabilitation of movements with the muscles in relaxed position, some experiments were conducted on human specimens in the dissections room of the Faculty of Medicine. This experimentation was necessary to calibrate the robotic platform. The efforts measured are always below $2.5 \mathrm{~kg}$ (Figure 5).

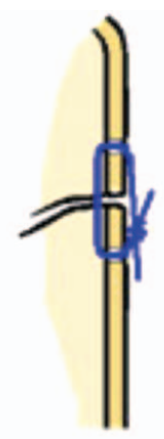

(a)
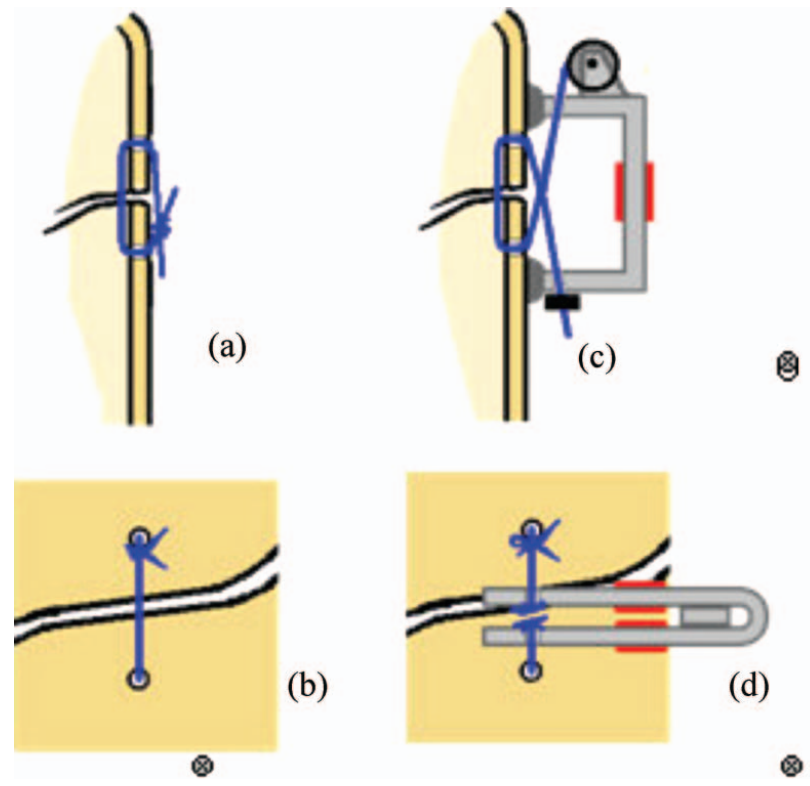

Figure 4. Subjecting method. (a) and (b) normal sewing, (c) bridge for adjusting and monitoring the suturing thread strain measurement and (d) measuring grip. 


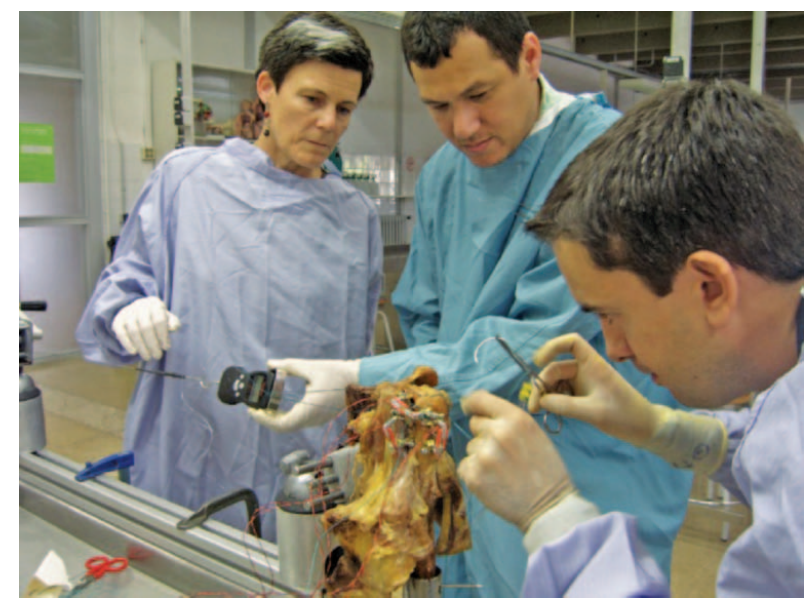

Figure 5. Monitoring the efforts on the suturing threads in tests with human specimens aimed to compare the measurements acquired with the workstation with respect to real operations.

Referring to bone consolidation studies, several devices are used to locate objects in a 3D space, optical devices and magnetic trackers being most common. If there are no ferromagnetic elements in the vicinity that can alter the magnetic field, magnetic trackers are very valuable due to their differential accuracy (sub-millimetre precision) and are not sensitive to occlusions. The differential accuracy permits a good bone fragment-to-fragment displacement monitoring.

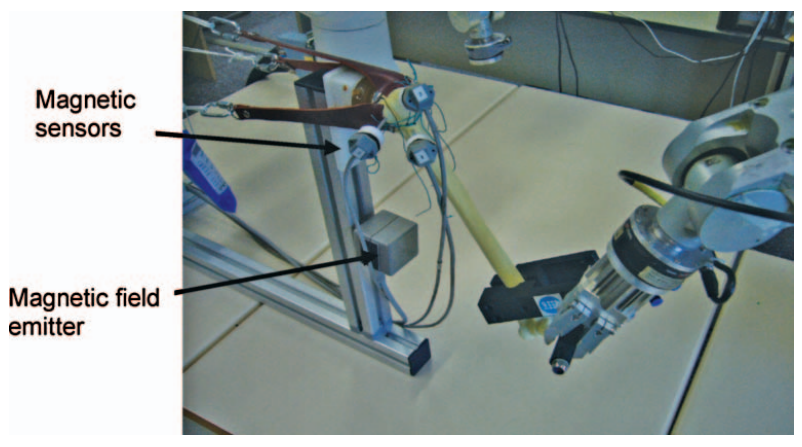

Figure 6. Robotic station with an artificial shoulder joint with magnetic sensor on the bone.

Due to the good performance of magnetic sensors, this technology is selected for this experimental platform. Figure 6 shows three sensors mounted on a bone to measure their relative displacements.

Figure 7 shows the distance between three magnetic trackers placed in three bone fragments of a humeral head during a simulated rehabilitation exercise, repeated four times. D12 represents (in tenths of millimetre) the relative distance between sensor 1 and sensor 2, and similarly D23 and D31.

The first trial process of the proposed robot environment has been described by Casals et al. (2008). In this experimentation process, the measures and monitoring on

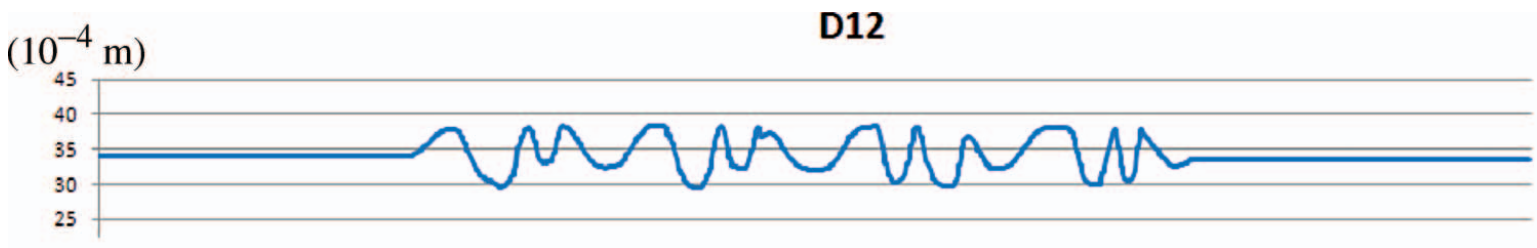

D23

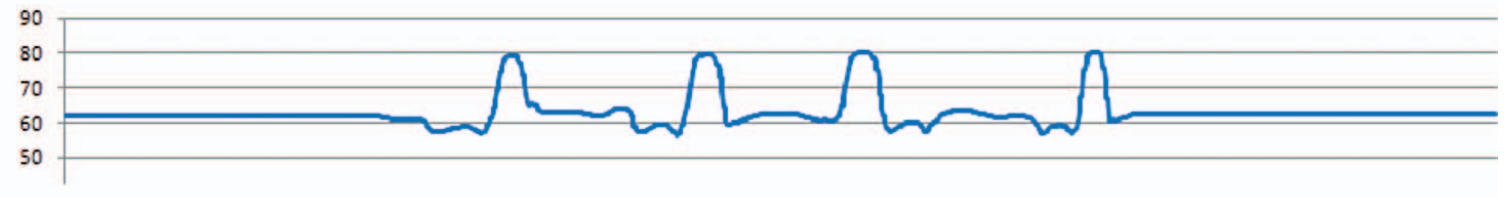

\section{D31}

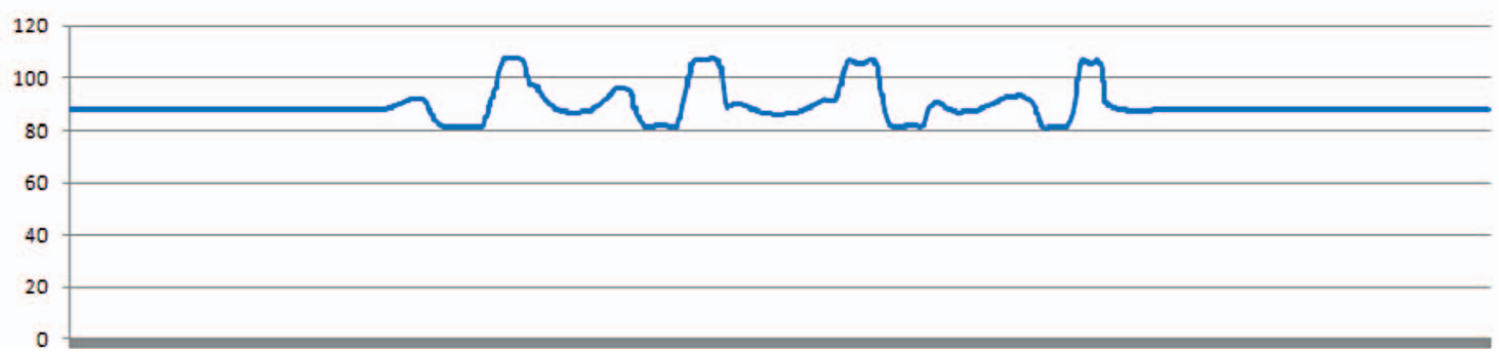

Figure 7. Measured distances between three bone fragments during a simulated rehabilitation exercise which is repeated four times (D12: distance between sensors 1 and 2, D23: distance between sensors 2 and 3, D31: distance between sensors 3 and 1). 
human specimens were used to estimate the range of forces involved in shoulder movements to reproduce a robotic station that works accordingly. Then a robot systematically repeats a set of programmed exercises over identical artificial bones, thus reproducible and comparable. This more objective and comparative strategy has been experimented with to compare different surgical techniques and procedures.

Other techniques developed for this workstation are related to teleoperation aids and specific human robot interaction techniques, movements controlled within virtual constraints or sliding over virtual surfaces.

\subsection{Objective evaluation criteria}

Due to the need for available objective evaluation tools when using the developed workstation, some computing tools have also been implemented. Therefore, from the signals provided by different sensors or measuring devices, these computing tools should provide a reference value that is a function of the measured variables. These variables, as for instance the displacements shown in Figure 7, can become valuable information for comparing different surgical suturing techniques.

In order to compare different techniques from such signals, it is necessary to obtain a final set of numerical descriptors. Here, one of the numerical descriptors used is the weighted sum $(S)$ of the root mean squared (RMS) value of each displacement signal during the exercise period $T$, and shown as

$$
S=\frac{1}{T} \sum_{\forall i, j}\left(\omega_{i j} \cdot \int_{0}^{T} d_{i j}^{2}(t) d t\right),
$$

where $d_{i j}$ are the A.C. values of the measured parameter, $d t$ is the distance between sensor $i$ and sensor $j$ and $\omega_{i j}$ are the weights $\left(\sum \omega_{i j}=1\right)$ that permit balancing the importance of one particular bone fragment stability in relation to others. An equivalent equation can be applied to force, $F(T)$. In this way, by selecting adequate weights, surgeons can give more relevance to a particular fragment, i.e. due to its size or due to its associated tendon.

Another very important set of numerical descriptors used here are the maximum displacement values reached for each pair of sensors during a period of time on a bone in movement, or maximum forces. Nevertheless, maximum values are also used as a threshold for discarding or not as a suture technique due to unacceptable displacement levels.

\section{Application to the improvement of humerus surgical arthroplasty}

Proximal humeral fractures are common among elderly people. Their incidence is dramatically increasing because

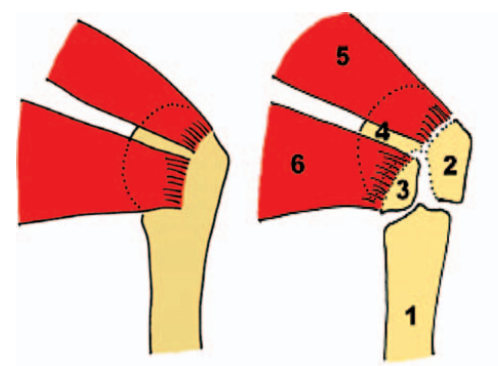

(a)
1. Humeral shaft

2. Greater tuberosity

3. Lesser tuberosity

4. Humeral head

5. Supraspinatus tendon

6. Subescapular tendon

(b)
Figure 8. Settling of the supraspinatus and infraspinatus muscles in the humeral head: (a) normal, (b) with a quadruple fracture.

of the presence of osteoporosis in such an old population. In addition, osteoporotic bones favour the presence of more complex patterns of fractures with no easy treatment and limited outcome.

In the most complex cases of proximal humeral fractures treatment with a prosthesis is mandatory. Patients treated with a prosthesis because of complex humeral fractures can expect good pain relief but functional outcomes are unpredictable.

The normal shoulder joint balances stability and movement to achieve the great range of motion experienced in the shoulder; this is the reason why the curvature radii of both joint surfaces are so different. To obtain stability, the tendinous complex, called the rotator cuff, is responsible for keeping the humeral head applied to the glenoid surface to allow deltoid muscle actuation to raise the arm. The junction of the supraspinatus, subscapular, infraspinatus and teres minor muscles forms the rotator cuff complex. The supraspinatus, infraspinatus and teres minor are attached to the greater tuberosity, while the subscapular is attached to the lesser tuberosity. When the proximal humerus breaks,

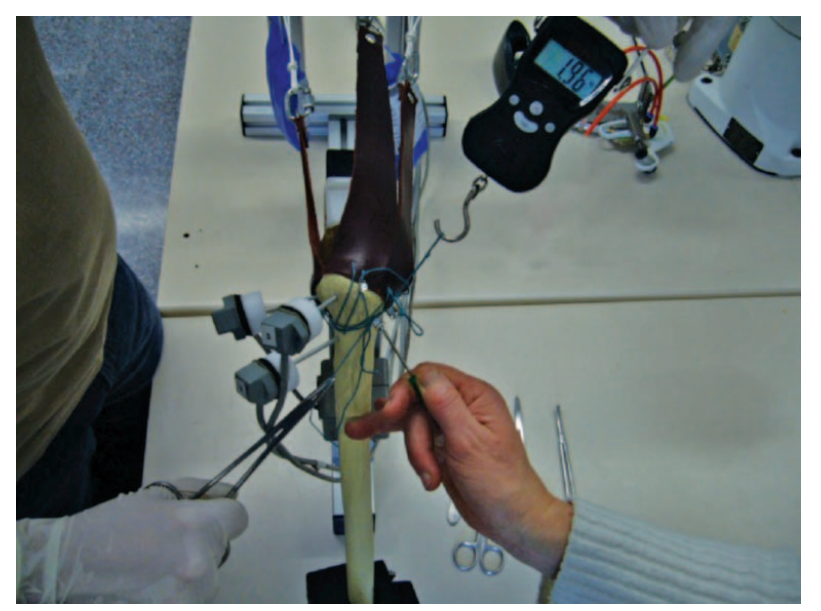

Figure 9. Procedure for the implementation of sutures with calibrated tensions. 
both tuberosities may pull apart, being displaced by the muscles attached to them. Figure 8 shows the settling of muscles and bone fragments in a quadruple fracture while Figure 9 shows the robotic support and measuring components for calibrating tensions in the proposed workstation.

Failure to restore the positions of the tuberosities and muscular attachments leads to poor functional results because of the lack of proper stability in the joint. Proximal humeral fractures, when treated with a prosthesis substitution, need the proper restoration and consolidation of the tuberosities to obtain a good functional outcome. Although surgical proper restoration and consolidation of the tuberosities depends on the fracture pattern and condition of osteoporosis in the bone, it mainly depends on the correct surgical technique. When the proximal humerus breaks, the main broken pieces are the humeral head, greater and lesser tuberosity and the humeral shaft, making it difficult to decide the correct height and version to which the prosthesis have to be implanted. The determination of the right height and version is a key issue in humerus arthroplasty.

Unfortunately, there is no good anatomical landmark to decide how high or low the prosthesis has to be implanted. There is no good landmark to decide the correct version to implant the prosthesis inside the humeral shaft. The correct positioning of the prosthesis is critical because the greater and the lesser tuberosities have to be reattached to the back of the prosthesis and have to be consolidated in the correct position to obtain a good functional outcome. For this reason some research is going on to improve this kind of surgery by finding ways to solve this uncertainty.

Following the work of Neer (1970), who started measuring displacements between tuberosities, Boileau and Walch (1997) studied the 3D geometry of the proximal humerus using a metrological instrument, and from these data the 3D shape was reconstructed to learn how to determine the best parameters: inclination, retroversion and medial and posterior offset to design an adequate prosthesis that fits with the shoulder anatomy. Frankle and Mighell (2004) addressed a study on landmarks based on anatomical geometry and external guides to perform a biomechanical and clinical evaluation of hemiarthroplasty. The hypothesis of the study was that different heights and versions of implantation might determine different forces passing through the sutures that fix the tuberosities in the back of the prosthesis. In Debski et al. (1995), a specific apparatus that parametrically emulates the human anatomy is described, showing that reproducible experiments, and thus a comparative analysis, can be done. In these trials, a study was done on the function of shoulder musculature and capsuloligamentous structures.

With this purpose a cadaveric static model and a robotic dynamic model were constructed (Frankle et al. 2002). Tension through the sutures between both tuberosities, between the greater tuberosity and the humeral shaft, and between the lesser tuberosity and the humeral shaft were monitored at different prosthesis heights and versions to determine the influence of these variations in the registered tensions. A big problem with these experiments with comparative purposes was the use of cadavers, which by nature show different characteristics, mainly in the conditions described, i.e. in people between 32 and 72 years old. The dispersion of bone characteristics makes any comparison of the measured results highly unreliable, thus becoming scientifically unrealistic.

\subsection{Surgical techniques}

The main problem in shoulder arthroplasty is that the bone portions should be fixed before getting welded, thus making sure that the patient performs rehabilitation exercises in order to avoid ankilosis. Considering the parameters that characterise shoulder arthorplasty, several measuring techniques based on specific machines or robotic systems have been developed.

In a first technique, bone fragments are united by sewing the suture threads through holes drilled in the bone, producing their coaptation. This technique, although mechanically correct, stumbles with the weakening problem due to the drilling.

A second technique, which tends to minimise the number of drilled holes, performs surrounding sutures that wrap the whole set of tuberosities. The drawback of this technique is the need to overcome tendons that can interfere with the desired thread path.

Therefore, it is convenient to establish mixed techniques that optimise the results using either method.

\subsection{The experimental station and testing procedure}

The procedure followed to achieve a repetitive and comparative evaluation of different surgical techniques consists of the following several steps:

1. Automatised cutting of identical artificial bones with identical fractures.

2. The execution of three types of sutures to be compared, operating in the same conditions using the developed platform that emulates a human shoulder joint.

3. Program some rehabilitation exercises with one of the available robots; these are repeated with the bones that have been sewed with three different techniques. The measures of suture strains and displacement of tuberosities are monitored during the execution of exercises.

4. Based on the above-defined evaluation criteria, the optimal strategy is selected.

\section{Suturing techniques evaluation}

The evaluation of the surgical technique that uses the described experimental station, and a further computing of 


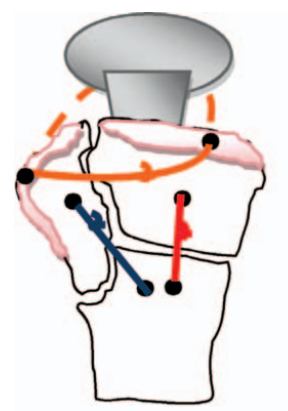

(a)

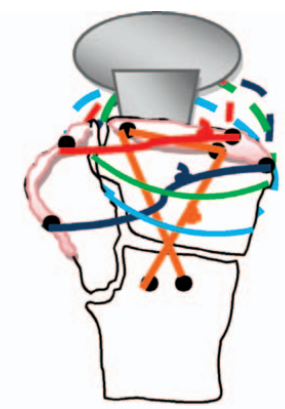

(b)

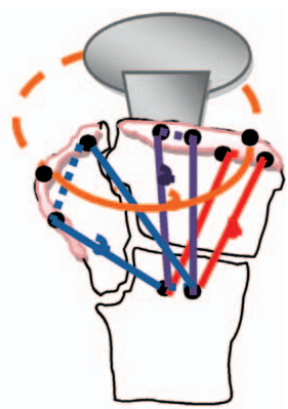

(c)

Figure 10. The three suturing techniques under evaluation.

the corresponding numerical descriptors, provide an objective quantification of the level of quality of different suturing techniques used to accommodate and stabilise the tuberosities with the implanted prosthesis. A correct suturing technique produces minimal displacement, which is fundamental to preserve vascularisation and fracture healing.

For the static model, which represents the situation of the arm being immobilised after surgery, the worst situation occurred when the prosthesis was placed in a lower and more retroverted position, and the suture at higher risk in this situation was the one joining both tuberosities. On the other hand, in the dynamic model, which represents patients undergoing an early rehabilitation program, the worst situation encountered took place when the prosthesis was placed in a lower and more retroverted position, but in this particular situation the suture at higher risk was the one joining the greater tuberosity and the humeral shaft.

For clinical practice it is very helpful to know how different positioning of the prosthesis changes the tensions through the sutures joining tuberosities because the proper positioning and healing of the tuberosities is mandatory to expect a good functional outcome. Thus, with the results obtained, it is recommended to avoid lower and retroverted placing of the prosthesis. In case surgeons fail to set the prosthesis at the proper height and version, special care has to be taken with suturing between the greater tuberosity and the humeral shaft if an early rehabilitation program is planned.

This evaluation process has been done considering three different suturing techniques to be able to compare their performances, referring to their capability to keep the fragments in a stable position during the joint movement.

The first proposed technique, considered as the simplest intervention, consists of three sutures: the first links the lesser tuberosity with the shaft head, the second links the greater tuberosity with the diaphysis and the third wraps the lesser tuberosity, the greater and the prosthesis, through its lateral fin (Figure 10a). The first two sutures require a bony perforation in each bone part to close the thread loop.
The second evaluated technique requires a more complex procedure because perforations of bone fragments have to be avoided in order to preserve bones' quality and their healing capability. This technique implies the use of five suturing threads. One of them joints the greater tuberosity and the diaphysis by means of a crossing loop through the supraespinatus tendon. Two of them embrace the tuberosities and the prosthesis through the medial side of the stem and the supraespinous and the subescapular tendons (Figure 10b). The last two tendons embrace the prosthesis with the greater tuberosity.

The third evaluated technique was performed without drilling tuberosities but with the aim of improving stability. We used the concept of tension-band wiring to take advantage of forces. It is composed of four suturing threads. The first attaches the lesser tuberosity with the diaphysis using the tendon-bone interface. The second and the third sutures put together the greater tuberosity and the diaphysis through the supraespinous tendon. The fourth thread embraces both tuberositis and the prosthesis (Figure 10c).

The analysis of these suturing techniques has also provided some cues for the better designing of prostheses, which is an advanced work aiming to facilitate a more effective mechanical joining effect for the suturing threads. Lower and more retroverted prosthesis positioning should always be avoided. In case of a wrong positioning of the prostheses, the surgeon must consider reinforcing the suture between both tuberosities if a static rehabilitation program is planned. On the other hand the suture between the greater tuberosity and the humeral shaft has to be reinforced when dealing with a dynamic rehabilitation program. As far as stability is concerned, the second and the third techniques studied are more reliable, but further investigation is required to determine better implementation for each technique.

\section{Conclusions}

With the goal of providing surgeons with a tool to evaluate surgical procedures and with a training station that 
implies a physical interaction when manual dexterity is an issue, a polyvalent robotic platform has been developed. The robotic workstation consists of two robots, aimed to execute programmed, teleoperated or autonomous tasks, as well as some auxiliary elements and sensors to emulate surgical procedures.

The processing of the sensor information provides an objective quantification of a quality parameter, which has proved to be effective to extract conclusions on some parameters that have an acceptance on surgical outcomes. Thanks to this methodological process, a new variant of surgical procedures now followed up in shoulder arthroplasty has been identified.

\section{References}

Boileau P, Walch G. 1997, Sep. The three-dimensional geometry of the proximal humerus. Implication for surgical technique and prosthesis design. J Bone Joint Surg Am. 79B(5):857865.

Casals A, Amat J, Frigola M, Rodriguez-Cheu LE, Torrens C, Ginés A. 2008. Monitoring and robotizing shoulder arthroplasty for training and optimization of suturing techniques. Int J CARS. 3(1-2):61-67.

Debski RE, McMahon PJ, Thompson WO, Woo SL-Y, Warner JP, Fu FH. 1995. A new dynamic testing apparatus to study glenohumeral joint motion. J Biomech. 28(7):869-874.

Frankle MA, Mighell MA. 2004. Techniques and principles of tuberosity fixation for proximal humeral fractures treated with hemioarthroplasty. J Shoulder Elbow Surg. 13(2):239247.

Frankle MA, Ondrovich LE, Markee BA, Harris ML, Lee WE. 2002. Stability of tuberosity reattachment in proximal humeral hemiarthroplasty. J Shoulder Elbow Surg. 11(5):413420.

Fuhan Shi H, Payandeh S. 2007. Real-time knotting and unkotting. Proceedings of the IEEE International Conference on Robotics and Automation, Rome, Italy, 10-14 April 2007.

Kiriyama Y, Matsumoto H, Imanishi N, Aiso S, Nakamura T, Toyama Y, Nagura T. 2008. Development of a miniature tension sensor to monitor suture tension during the joint motion. J Biomech. 41(503).
Mizuuchi I, Tajima R, Yoshikai T, Sato D, Nagashima K, Inaba M, Kuniyoshi Y, Inoue H. 2002, Oct. The design and control of the flexible spine of a fully tendon-driven humanoid, Kenta. Proceedings of the 2002 IEEE/RSJ international conference on intelligent robots and systems, Lausanne, Switzerland, vol 3, pp 2527-2532.

Neer CS, 1970. Displaced proximal humerus fractures, I and II. J Bone Joint Surgery, AM.

Nishikawa K, et al. 2003, Oct. Modeling and Analysis of Elastic Tongue Mechanism of Talking Robot for Acoustic Simulation IEEE/RSJ International Conference on Intelligent Robots and Systems.

Rosen J, Brown JD, Chang L, Barreca M, Sinanan M, Hannaford B. 2002. The BlueDRAGON - a system for measuring the kinematics and the dynamics of minimally invasive surgical tools in-vivo. Proceedings of the IEEE International Conference on Robotics \& Automation.

Schubert HM, Moser TM, Buchegger JW, Brodbeck AF, Schoeller T, Zimmermann RF, Hohlrieder M. 2006. Tyrolean tensiometer: A new instrument for easy intraoperative tension measurement before nerve coaptation. J Trauma Inj, Infect and Crit Care. 61(3):760-763.

Solis J, Oshima N, Ishii H, Matsuoka N, Takanishi A, Hatake K. 2009. Quantitative assessment of the surgical training methods with the suture/ligature training system 'WKS-2RII'. Proceedings of IEEE International Conference on Robotics and Automation, 12-17 May 2009, Kobe, Japan.

Takanobu H, Yajima T, Nakazawa M, Takanishi A, Ohtsuki K, Ohnishi M. 1998, May. Quantification of masticatory efficiency with a mastication robot. Proceedings of IEEE International Conference on Robotics \& Automation, 16-20 May 1998, Leuven.

Wang M, et al. 2006. The design and implementation of virtual system for the robot-assisted setting-bone surgery. Proceedings of BioMicro and Nanosystems Conference, 15-18 January 2006, San Francisco, CA, USA.

$\mathrm{Xu}$ WL, Bronlund J, Kaiser J. 2005. Choosing new ways to chew: A robotic model of the human masticatory system for reproducing chewing behaviors. IEEE Robot Autom Mag 12(2):90100.

Zahraee AH, Szewczyk J, Morel G. 2009. Simulation for optimal design of hand-held surgical robots. Paper presented at: EMBC 2009. Proceedings of the IEEE Engineering in Medicine and Biology Society Annual International Conference; 2009 Sep 3-6; Minneapolis, MN. 

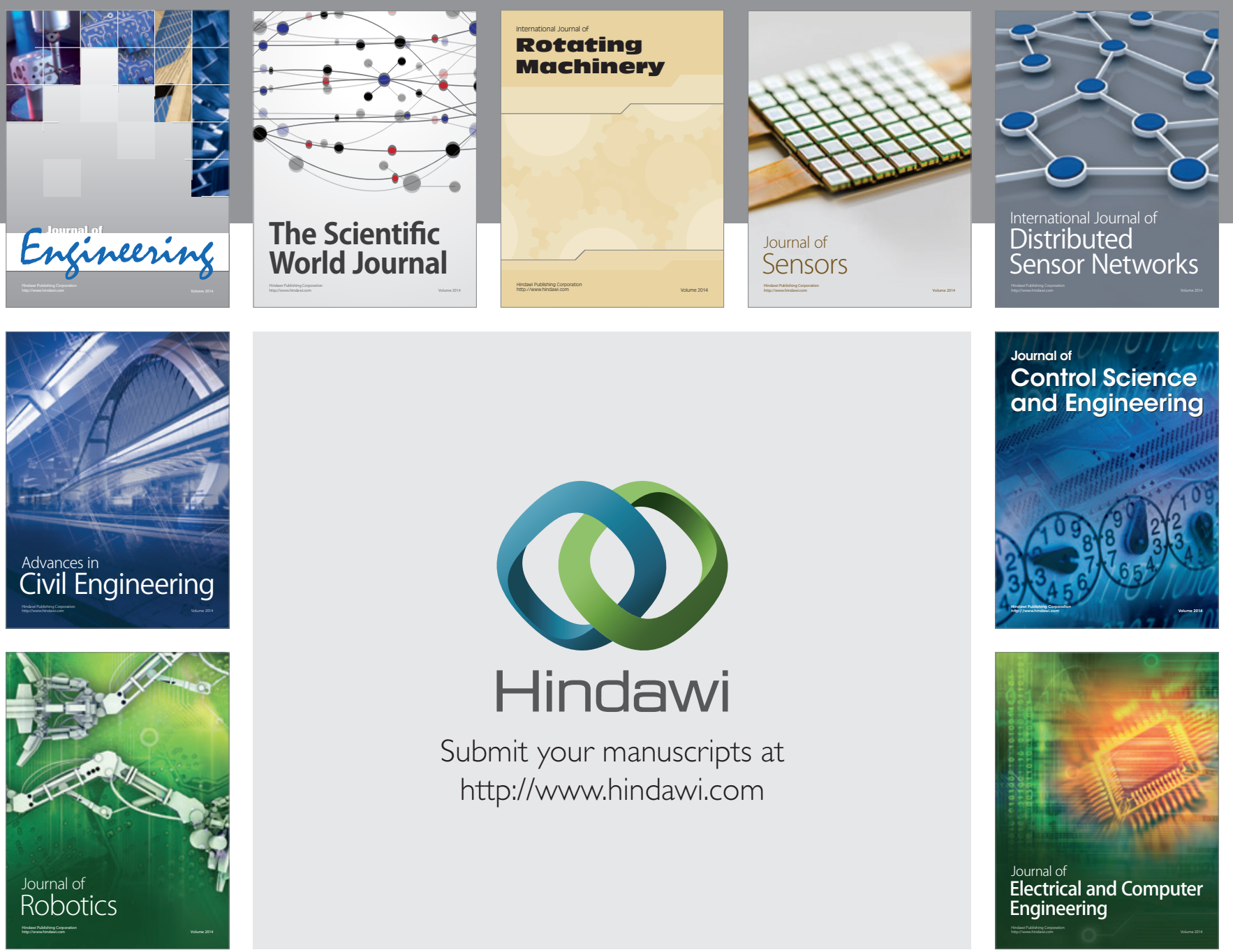

Submit your manuscripts at

http://www.hindawi.com
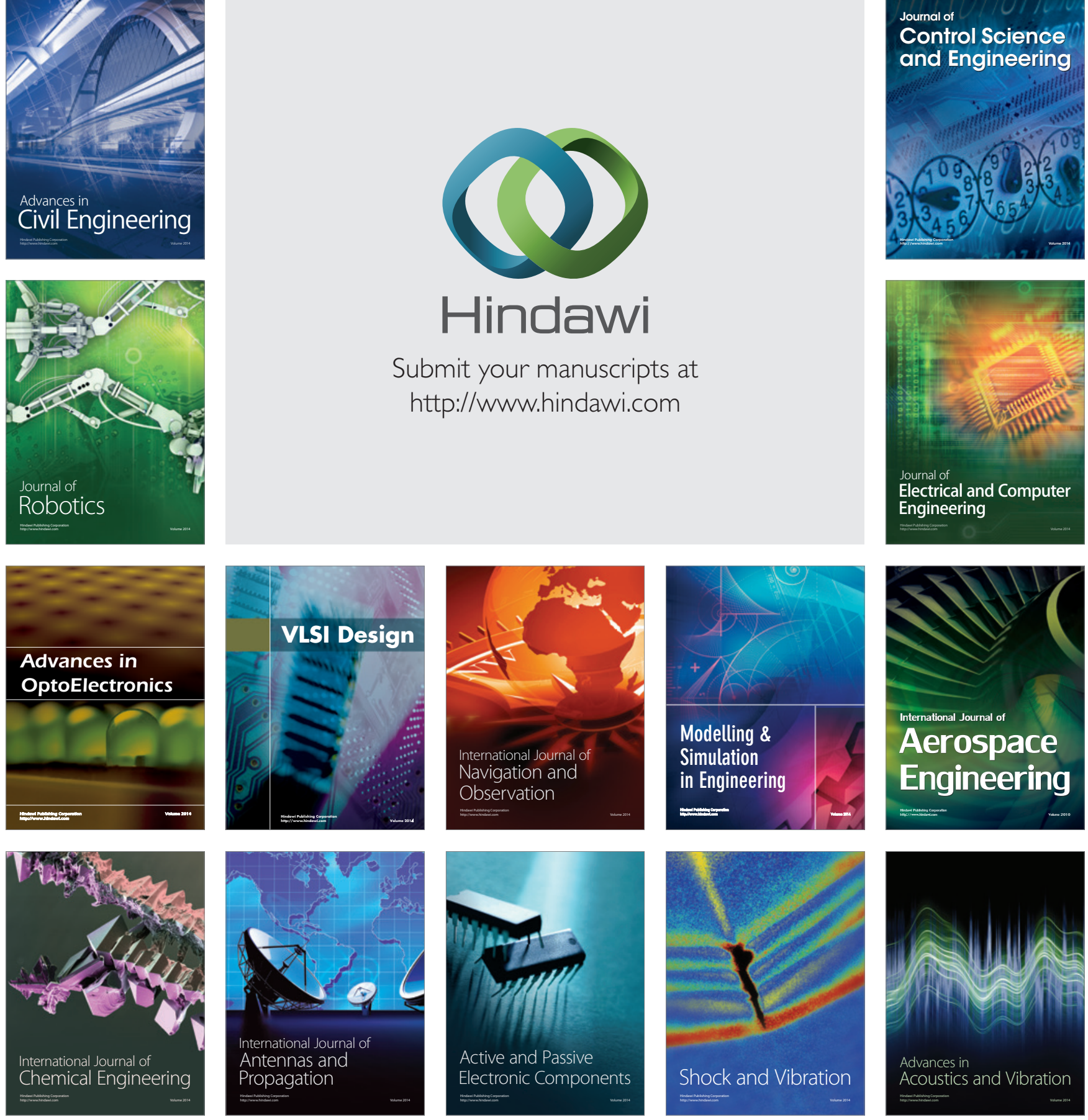